remission. The potential for immunosuppression to increase the risk of opportunistic infection was recognised early with the publication of ECCO Consensus guidelines on prevention diagnosis and management of opportunistic infections in IBD. Has this permeated to the grass roots of clinical care or are we still "top-heavy"? We reviewed vaccination practices in a large primary care cohort.

Methods Data was obtained from primary care in the Fylde and Wyre CCG from electronic patient records maintained on EMIS Web. Patients were considered immunosuppressed if they were on doses of prednisolone $>20 \mathrm{mg} /$ day or equivalent for 2 weeks or more, ongoing treatment with effective doses of Thiopurines, Methotrexate, Infliximab and Adalimumab or had these agents discontinued within 3 months. We identified patients treated with these drugs between September 2012 and August 2013. Vaccination practices were reviewed in line with ECCO consensus. Data on biologic therapy and steroids was not available.

Results A total patient population of 93,240 (adult population of 75,952 ) from 13 practices was thus audited. A total of 594 patients were prescribed IM therapy [Azathioprine (AZA) 135, Methotrexate (MTX) 446, 6MP 13]. The data on vaccinations is shown in Table 1 . Vaccination rates were significantly higher in the non IBD cohort as compared to the IBD cohort, for influenza vaccine $\mathrm{p}<0.001,95 \% \mathrm{CI}(-0.17 \pm 0.1)$ and pneumococcal vaccine $\mathrm{P}<0.00195 \%$ CI $(-0.177 \pm 0.103)$.

Conclusion The practice of immunisation was poor and probably reflective of disparate practices elsewhere. Mere existence of guidelines is insufficient for quality improvement, which must take into account and remedy barriers to implementation. These include clinician awareness, concerns regarding safety and side effects and ambiguity regarding responsibility. Gastroenterologists must provide a clear line of communication to primary care physicians. We are taking necessary steps through a dedicated proforma to ensure vaccination uptake.

Disclosure of Interest None Declared.

\section{PTU-061 INFLIXIMAB OR CICLOSPORIN: PATIENTS' TREATMENT PREFERENCES AND THE IMPACT OF ULCERATIVE COLITIS (UC) ON THEIR LIVES}

A Seagrove ${ }^{*}$, F Rapport, I Williams. Swansea University, Swansea, UK

10.1136/gutjnl-2014-307263.135

Introduction The qualitative element of CONSTRUCT, a randomised controlled trial comparing clinical and cost effectiveness of infliximab and ciclosporin in steroid resistant UC, contributed to the specific trial objectives of examining quality of life (QoL) across the two groups. The qualitative element concentrated on patient and professional perceptions, and we describe patients' opinions about treatments and other UC therapies.

Methods Semi-structured telephone interviews with patients three and 12 months after admission with acute severe UC and randomisation. Thematic analysis was conducted by three qualitative researchers, followed by group analysis by seven members of CONSTRUCT. This abstract concentrates on the three month data.

Results 20 interviews were completed. Length of disease duration varied but similar stories emerged about living and coping with UC, the physical, mental and emotional impact of the disease, treatment options and concerns and hopes for the future. The main issues were:
- Patients favour infliximab because they perceive a more positive treatment outcome, easier treatment regime and fewer side effects

- The dramatically debilitating symptoms that impact on patients' QoL, their family and friends, are particularly noticeable in this disease

- Patients live with the ongoing unpredictability of symptoms and treatment, making it particularly difficult for patients and healthcare professionals to manage care

- Unlike other chronic diseases, UC is considered embarrassing, making it an isolating and awkward experience for patients and difficult to manage work and life routines

- The lack of visibility of symptoms or outcomes impacts on patients' ability to share and discuss openly with others

- Surgery is feared but most patients experience relief and subsequent recognition of health benefits following surgery

- Patients would like to understand what causes UC, its links to stressors and diet and would welcome more extensive information provision

- Ready access to IBD Nurses was considered important.

Conclusion Study findings indicate that UC patients live with constant, unpredictable symptoms, where a flare-up becomes socially isolating along with anxieties of deteriorating health. Patients need support to manage the impact of UC on their lives, consider prompt diagnosis to be important, and need relevant treatment provided quickly. Patients clearly prefer infliximab because of the easier treatment regime and fewer side effects. The views of patients after surgery were generally positive, but more research is required into surgical treatment of UC to support those facing surgery and as an alternative to medical treatment. The profile of UC should be raised to destigmatise the disease and thus the embarrassment felt by sufferers and those living with the outcomes of surgery.

Disclosure of Interest None Declared.

\section{PTU-062 CROHN'S DISEASE-ASSOCIATED ESCHERICHIA COLI CAN TOLERATE LOW NUTRIENT, LOW PH AND HIGH CHEMICAL STRESS ENVIRONMENTS ENCOUNTERED WITHIN THE PHAGOLYSOSOME OF MUCOSAL MACROPHAGES}

A Tawfik*, JM Rhodes, BJ Campbell. Gastroenterology, Institute of Translational Medicine, Liverpool, UK

\subsection{6/gutjnl-2014-307263.136}

Introduction Mucosa-associated adherent, invasive E.coli (AIEC), found in increased number in Crohn disease (CD) ileal and colonic mucosa, can replicate within host mucosal macrophages $^{1,2}$ Other pathogens, known to survive within macrophages (e.g. Mycobacteria and Salmonella), possess stressresponse inducible systems that allow them to tolerate environmental stress. Here we aimed to establish whether CD AIEC are better able than non-AIEC E.coli to tolerate low $\mathrm{pH}$, high nitrosative and oxidative stress conditions, that mimic the environment within the macrophage phagolysosome.

Methods CD AIEC isolates possessing ability to replicate intracellularly in J774-A1 macrophages (ileal isolate LF82 and 3 colonic isolates HM427, HM605 and HM615; range 4.5 to 9fold replication) were compared to E.coli strains killed by macrophages (XL1 and EPI300). Bacterial cultures were grown at $37^{\circ} \mathrm{C}$ in Luria-Bertani (LB) medium to $\mathrm{OD}_{600 \mathrm{~nm}} 0.1$ and diluted in 10 -fold steps in $0.9 \% \mathrm{w} / \mathrm{v}$ saline. Samples were spotted onto LB agar containing one of the following stress agents: $100 \mathrm{mM}$ 4-Morpholine ethanesulfonic acid (MES) pH5, 100mM MES 
$\mathrm{pH} 5+1 \mathrm{mM} \mathrm{NaNO}_{2}$ (high nitrosative stress), $1 \mathrm{mM} \mathrm{H} \mathrm{H}_{2} \mathrm{pH} 7$ (high oxidative stress) and $1 \mathrm{mM}$ methyl viologen (MV) $\mathrm{pH} 7$ (super oxidative stress). Plates were incubated overnight at $37^{\circ} \mathrm{C}$. Results All four CD AIEC showed tolerance to low $\mathrm{pH}$, high nitrosative and oxidative stress mimicking the intra-phagolysosome environment. CD AIEC demonstrated greater tolerance to growth at $\mathrm{pH} 5$. Compared to growth seen on LB agar $\mathrm{pH} 7$ (100\%), LF82 showed $99.3 \pm 22.2 \%$ growth at pH5 [mean \pm SD]. Likewise, growth of HM427 (104.4 \pm 15.2\%), HM605 $(81.1 \pm 15.5 \%)$ and HM615 $(94.2 \pm 9.4 \%)$ was also seen at pH5. Conversely, $\%$ growth seen for laboratory strains was only $64.2 \pm 9.9 \%$ for XL-1 and $61.3 \pm 8.7 \%$ for EPI300; $\mathrm{N}=4$ expts, $\mathrm{n}=3$ replicates $(\mathrm{P}<0.01$; ANOVA). Most remarkable was tolerance on super oxidative LB agar containing $1 \mathrm{mM} \mathrm{MV}$, with LF82 showing growth at $95.9 \pm 11.5 \%$, HM427 $81.3 \pm$ 17.9\%, HM615 $102.4 \pm 14.3 \%$ and HM605 $86.1 \pm 13.5 \%$ vs. that seen on standard LB agar (100\%). Non-AIEC strains showed little tolerance to all stress conditions tested (in $1 \mathrm{mM}$ MV; XL-1, no growth; EPI300, $1.8 \pm 1.2 \%$ growth vs. LB agar alone, 100\%); $(\mathrm{P}<0.0001)$. The four $\mathrm{CD}$ AIEC were also observed to grow better after $8 \mathrm{~h}$ in a low nutrient M9 medium supplemented $0.1 \%$ casamino acids $(\mathrm{pH} 4)$ than the laboratory $E$. coli tested and 3 additional E.coli isolates from healthy controls (ECOR1, ECOR35, ECOR51); $\mathrm{N}=1, \mathrm{n}=3$.

Conclusion Crohn's AIEC, unlike non-AIEC E. coli, tolerate low nutrient, low $\mathrm{pH}$ and high chemical stress conditions that mimic the macrophage phagolysosome environment.

\section{REFERENCES}

1 Bringer MA, et al. Cell. Microbiol 2006;8:471-84.

2 Subramanian S, et al. Antimicrob. Agents Chemother 2008;52:427-34.

Disclosure of Interest A. Tawfik: None Declared, J. Rhodes Grant/research support from: Provexis plc., Consultant for: advisory boards for Atlantic, Procter and Gamble and Falk, Speaker bureau with: honoraria from Abbott, Falk, Ferring, Glaxo Smith Kline, Procter and Gamble and Schering Plough, Conflict with: the University of Liverpool and Provexis plc, holds a patent for use of a soluble fibre preparation as maintenance therapy for Crohn's disease, B. Campbell Grant/research support from: Provexis plc, The Bo and Vera Axe:son Johnson Foundation for Nature Medicine Ltd and Arcis Biotechnology, Speaker bureau with: honorarium from Amgen Inc.

\section{PTU-063 A PROSPECTIVE AUDIT OF THE USE OF TACROLIMUS IN PATIENTS WITH REFRACTORY SUBACUTE ULCERATIVE COLITIS IN A DISTRICT GENERAL HOSPITAL}

AS Dhillon*, AW Harris. Department of Gastroenterology, Tunbridge Wells Hospital, Kent, UK

\subsection{6/gutjnl-2014-307263.137}

Introduction Tacrolimus appears to be effective short-term treatment for patients with refractory ulcerative colitis (UC) $)^{1}$ and is recommended by $\mathrm{NICE}^{2}$ in suitable patients. We report our experience in a district general hospital out-patient setting of tacrolimus in patients with steroid refractory subacute UC whom either failed, or were intolerant to thiopurines and as an alternate to surgery. In England, Wales and Northern Ireland infliximab may no longer be used to treat patients with sub-acute UC. ${ }^{3}$ Methods A prospective quality and safely assurance audit was undertaken of all patients with UC treated with tacrolimus from January 2010 until January 2014. Patients not responding to or intolerant of conventional therapy met with the consultant gastroenterologist (AWH). They were offered treatment with tacrolimus, provided with written drug information or referral for surgical management. All agreed to start treatment with tacrolimus (Prograf) $0.1 \mathrm{mg} / \mathrm{kg} /$ day in 2 divided doses and were monitored according to local protocol with FBC, UandEs and serum trough levels at weeks 2, 4 and 3 monthly thereafter. The dose of tacrolimus was titrated aiming for serum trough levels between $5-20 \mathrm{ng} / \mathrm{mL}$. Clinical response was assessed by AWH in clinic.

Results Seventeen patients (8 female; mean age 38 [range 1986]) were treated with tacrolimus. Eleven patients (65\%) had a clinical response (median treatment duration 12 [range 2-192] weeks). Four of these $(45 \%)$ patients developed intolerance to tacrolimus (renal impairment $\mathrm{n}=2$; tremor $\mathrm{n}=1$; paraesthesia $\mathrm{n}=1$ ) and stopped treatment. Seven patients continued on tacrolimus with clinical response and without side effects. Of the 10 patients whom either failed to respond or were intolerant of tacrolimus, 7 underwent colectomy. Of the remaining 3 patients, 1 declined surgery and 2 patients have responded to treatment with methotrexate.

Conclusion Our experience supports the use of tacrolimus in patients with subacute UC refractory to conventional treatments as an alternative to elective surgery as infliximab is no longer recommended in these circumstances. ${ }^{3}$ However only 7 of 17 $(41 \%)$ patients had a clinical response and tolerated tacrolimus. Close monitoring of renal function and serum trough levels is required. The long-term efficacy and safety of tacrolimus remains unclear.

\section{REFERENCES}

1 Baumgart DC, MacDonald JK, Feagan B. Tacrolimus (FK506) for induction of remission in refractory ulcerative colitis. Cochrane Database of Systematic Reviews 2008, Issue 3. Art. No.: CD007216. DOI: 10.1002/14651858. CD007216

2 National Institute for Health and Care Excellence Ulcerative colitis: management in adults, children and young people CG 2013;166

3 National Institute for Health and Care Excellence Infliximab for subacute manifestations of ulcerative colitis TA 2008;140

Disclosure of Interest None Declared.

\section{PTU-064 INCREASED MUCOSAL EXPRESSION OF INSOLUBLE KERATINS 8, 18 AND 19 IN LONG-STANDING ULCERATIVE COLITIS IN COMPARISON TO RECENT-ONSET ULCERATIVE COLITIS: VALIDATION OF MASS SPECTROMETRY DATA}

${ }^{1}$ A Assadsangabi*, ${ }^{2} \mathrm{C}$ Evans, ${ }^{1} \mathrm{D}$ Majumdar, ${ }^{3} \mathrm{BM}$ Corfe, ${ }^{1} \mathrm{AJ}$ Lobo. ${ }^{1}$ Gastroenterology, Royal Hallamshire Hospital, UK; ${ }^{2}$ Chemical and Biological Engineering, University of Sheffield, Sheffield, UK; ${ }^{3}$ Oncology, University of Sheffield, Sheffield, UK

\subsection{6/gutjpl-2014-307263.138}

Introduction Intermediate filaments (IF) are one of the main components of the human cell cytoskeleton with keratins (K) being the largest component. K8, K18 and K19 constitute the main keratins in the intestinal epithelial cells. Keratin alteration may play a role in the pathophysiology of ulcerative colitis (UC). K8 -/- mice develop chronic colitis. K8 and K18 play a role in TNF- $\alpha$ induced-apoptosis. We have previously shown increased expression of insoluble K8, K18 and K19 in longstanding UC relative to recent - onset ulcerative colitis $(\leq 5$ years) using mass spectrometry (MS) in the IF fraction of pooled patient samples. The aim of this study was to use antibody-based relative quantification of K8, K18, K19 in individual patient samples to validate MS results and describe variation in expression across the cohort. 